\title{
Remote triggering of high voltage systems by laser-induced plasmas
}

\author{
N. J. West ${ }^{\mathrm{a}^{*}}$, I. R. Jandrell ${ }^{\mathrm{a}}$, A. Forbes ${ }^{\mathrm{b}, \mathrm{c}}$ \\ ${ }^{a}$ School of Electrical and Information Engineering, University of the Witwatersrand, Johannesburg \\ Private Bag 3, 2050 \\ ${ }^{\mathrm{b}}$ CSIR/NLC P.O. Box 395, Pretoria 0001, South Africa \\ ${ }^{\mathrm{c}}$ University of KwaZulu-Natal, Private Bag X54001, Durban 4000, South Africa
}

\begin{abstract}
The aim of this paper is to compare the electrical performance of an orthogonally with a coaxially laser-triggered spark gap. Each of these two gaps has its own advantages and disadvantages. At the same time, a Rogowski profile spark gap was investigated in terms of its orthogonally laser-triggered performance. It was found that the Nd:YAG laser used $(1064 \mathrm{~nm}, 800 \mathrm{~mJ})$ was able to reduced the breakdown voltage of a $50 \mathrm{~mm}$ gap by $70 \%$ from $135 \mathrm{kV}$ to about $40 \mathrm{kV}$. The position of the laser-induced plasma was found to play a significant role in the breakdown process - best results being obtained when the laser was focused in the centre of the gap. Finally, the shape of the laser-induced arc is dependant on the applied electric field. When the field is low, the arc tends to avoid the laser-induced plasma thus exhibiting a very anomalous behaviour. When the field is increased, the arc tends to attach itself to the plasma as expected.
\end{abstract}

Keywords: Laser-induced plasma, laser-induced arc, electric field, breakdown probability

\section{INTRODUCTION}

The formation of a laser-induced plasma was first observed in 1963 by Maker, Terhume and Savage ${ }^{[1]}$. It was noted that a focused laser beam was able to generate a spark at the focal point of the lens. Since then, research has been performed in order to study this phenomenon and to find possible applications. Applications vary from laser gas ignition ${ }^{[2]}$ to lasertriggering of spark gaps ${ }^{[3-4]}$ and of late, lasers have been used in laser-triggered lightning experiments ${ }^{[5-6]}$

There are two main strategies for achieving laser-induced breakdown in a spark gap. These are the orthogonal and coaxial geometries. In the coaxial (and most common) geometry, the beam is focused along the length of the gap axis. This allows the laser beam to irradiate the entire gap. In the orthogonal approach, the beam is directed at right angles to the gap axis. Although this method is not intuitive, it has some important advantages over the coaxial approach. An orthogonal gap offers a more practical geometry, allowing the user to modify the gap length and gap type without worrying about directing the laser beam though one of the electrodes.

Breakdown in the case of an orthogonal gap can be thought of as a result of a severe modification of the electric field. The high beam intensities produced by the laser at the focal point of the lens leads to ionization of the gas in the gap a d formation of a laser-induced plasma. This introduces a disturbance to the applied steady state DC field across the gap, thus leading to electrical breakdown.

In this paper, experiments involving orthogonal gaps triggered by a high-power Nd:YAG laser are presented. Finally, a study of the spatial and temporal interaction of the laser-induced plasma and the laser-induced arc is presented.

\footnotetext{
**njwplasma@gmail.com; phone +27 11717 7258; fax +27 114031929
} 


\section{BACKGROUND}

One of the most important parameters that governs the phenomenon of laser-induced breakdown is the laser beam intensity. It is well known that a laser beam is able to cause breakdown in air if the intensity at the focal point is above a certain threshold ${ }^{[7]}$. The peak laser beam intensity can be given by the following well-known relationships:

$$
I_{0}=\frac{2 E_{T}}{\pi \omega_{f}^{2} \tau}
$$

with

$$
\omega_{f}=\frac{M^{2} \lambda f}{\pi \omega_{0}}
$$

Here $I_{0}$ is the peak laser beam intensity for a source of wavelength $\lambda, E_{T}$ is the total energy of each pulse, $\omega_{f}$ is the beam radius at the focal plane of the lens, $\omega_{0}$ is the beam radius incident on the lens (of focal length $f$ ) and $\tau$ is the FWHM laser time pulse. For Gaussian beams the beam quality factor, $M^{2}$, is equal to 1 , and greater than 1 for all other intensity profiles.

Another very important parameter that governs the laser-induced breakdown process is the wavelength of the laser beam used. It is known that a beam with a shorter wavelength will deliver higher energy photons to the focusing region than those of a longer wavelength. Also as seen above (Equation 2), a shorter wavelength beam will provide a tighter focus. However, it has been noted experimentally that the wavelength affects the overall breakdown process to a lesser extent than parameters such as the laser pulse width and energy ${ }^{[8]}$.

\section{EXPERIMENTAL SETUP}

The experimental setup can is shown in Figure 1. A Q-switched, flash-lamped pumped Nd:YAG laser (Continuum, Powerlite) operating at the fundamental wavelength of $1064 \mathrm{~nm}$ was used. The linearly polarised output beam could be varied in both energy and pulse width through adjustment of the Q-switch delay. The repetition rate of the laser was set at a fixed $10 \mathrm{~Hz}$ throughout the experiments. A visible Helium Neon laser was aligned to be collinear to the Nd:YAG laser for ease of optical alignment. The laser beam passed through two Brewster windows which could be rotated, thus allowing energy attenuation external to the laser cavity. A pop-up mirror allowed for the laser beam to be directed to laser diagnostics: laser pulse duration was measured with a fast Si photodiode (ThorLabs, model DET210) and an energy meter (Gentec, model ED200) was used to determined the total pulse energy.

With the pop-up mirror in the down position, the laser beam was delivered to a final focusing lens (uncoated quartz, plano-convex) for plasma generation. The spot size at the focus could be changed by changing the focal length of the lens.

The electrodes of the spark gap had a Rogowski profile and were arranged in such a way so as to allow the laser beam to intersect the gap axis at right angles. Rogowski profiles were used in order to create as uniform a field as possible in the spark gap. The vertical position of the focal point in the gap was varied by adjusting the height of the spark gap above the laser table. The gap was adjustable up to $50 \mathrm{~mm}$. A Spellman DC generator (max $50 \mathrm{kV}$ ) was used in these experiments to energize the gap.

In order to ensure that the camera did not saturate, the following procedure was performed: The brightest image of the plasma formation was determined and the camera gain reduced until the saturation was minimised. All further images were recorded at this gain setting. 


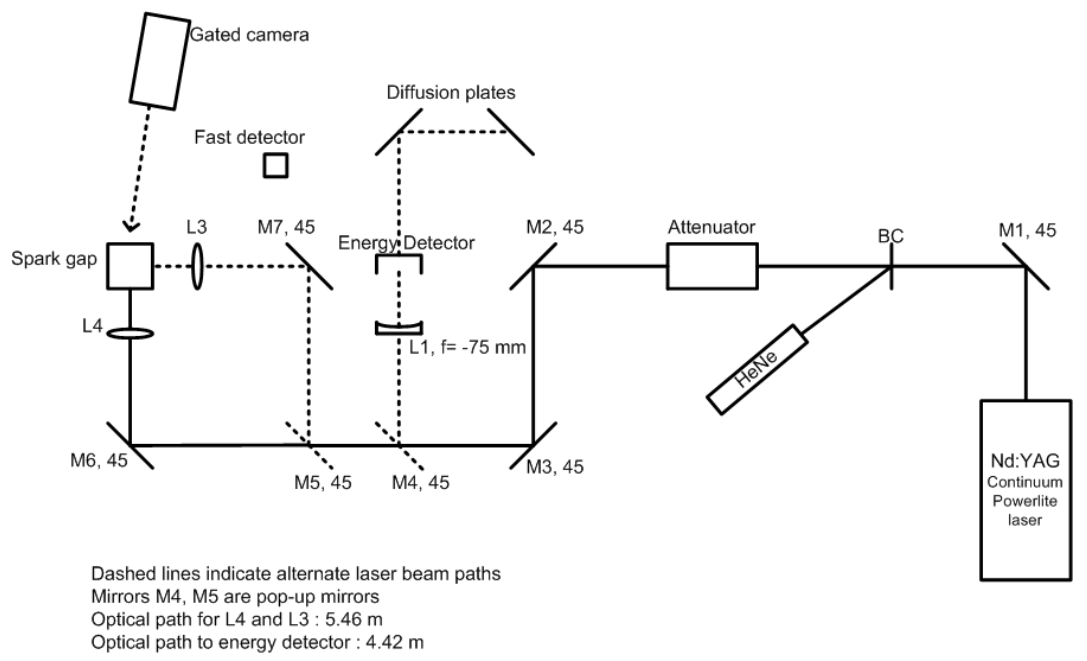

Fig. 1. Laser and optics setup for the Nd:YAG experiments

The time evolution of the laser-induced plasma was monitored with a gated camera (Xybion, model ISG-250 - later a Cooke Corporation FlashCam Pro camera was used) aligned to face along the laser beam axis, for "head-on" plasma recording, or perpendicular to the laser beam axis, for a side view of the plasma. The gate time of the camera was set to $50 \mathrm{~ns}$, and delayed relative to the laser pulse by means of a Stanford Delay Generator (model DG-535).

Typical orthogonal and coaxial geometries used in the experiments can be seen below in Figure 2.
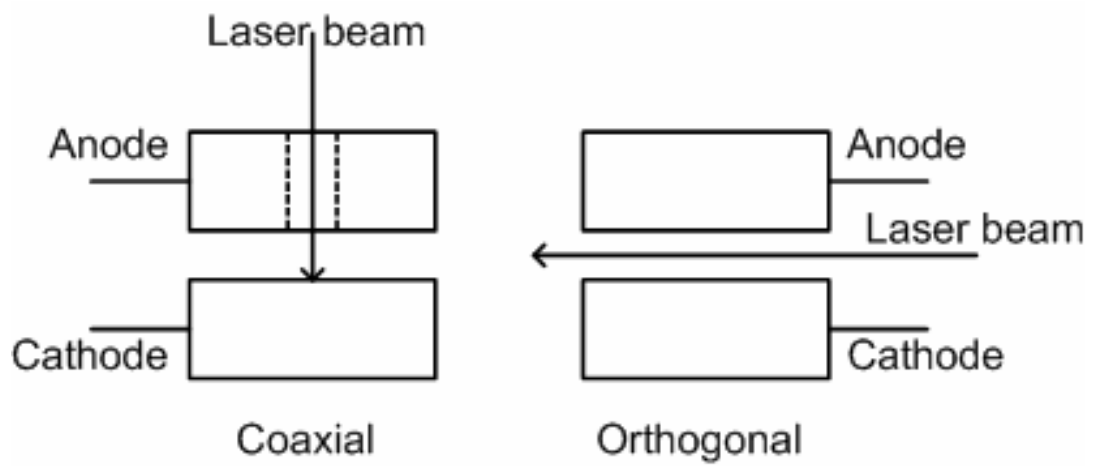

Fig. 2. Coaxial and orthogonal spark gap configurations used in the experiments.

\section{ORTHOGONAL VS. COAXIAL GAP RESULTS}

The first set of experiments, compared the 'traditional' coaxial spark gap arrangement with the orthogonal geometry. As mentioned, the coaxial geometry has been preferred over the orthogonal case since the laser is allowed to irradiate the entire length of the gap. In some cases, the beam was allowed to strike the surface of one of the electrodes allowing some of the electrode material to be ablated from the surface. This can help in the breakdown process since it allows foreign objects and charge carriers to be liberated and to assist in the breakdown process. The result is a reduced breakdown strength of the gap. Results such as these cannot be thought of as 'clean' since the results would depend on how easy the electrode surface is ablated. However, a coaxial gap is in many ways impractical. It is difficult to construct and not so easy to set up, since it involves aligning the laser very carefully through a hole that is often drilled through one (or both) of the electrodes. 
On the other hand, an orthogonal arrangement does not allow compete irradiation of the gap. The major advantage of such a configuration is that it is easily constructed and the setup time is far less since there is no need for careful beam alignment.

It is therefore interesting to compare these two configurations in terms of their breakdown performance. The aim of the experiments described in this section is to find the minimum voltage the laser can trigger $\mathrm{V}_{\mathrm{L}}$ and compare it to the maximum voltage the gap is able to withstand $\mathrm{V}_{\mathrm{W}}$. This can also be expressed in terms of a breakdown range $\Delta \mathrm{V}=\mathrm{V}_{\mathrm{W}^{-}}$ $\mathrm{V}_{\mathrm{L}}$.

The results of this experiment can be seen in Table 1 and 2 below:

Table 1. Coaxial gap breakdown voltages

\begin{tabular}{|c|c|c|c|}
\hline Gap length $[\mathbf{m m}]$ & $\mathbf{V}_{\mathbf{L}}[\mathbf{k V}]$ & $\mathbf{V}_{\mathbf{W}}[\mathbf{k V}]$ & $\mathbf{\Delta V}[\mathbf{k V}]$ \\
\hline 5 & 1.8 & 15 & 13.2 \\
\hline 10 & 6 & 27 & 21 \\
\hline
\end{tabular}

Table 2. Orthogonal gap breakdown voltages

\begin{tabular}{|c|c|c|c|}
\hline Gap length $[\mathbf{m m}]$ & $\mathbf{V}_{\mathbf{L}}[\mathbf{k V}]$ & $\mathbf{V}_{\mathbf{W}}[\mathbf{k V}]$ & $\mathbf{\Delta V}[\mathbf{k V}]$ \\
\hline 5 & 0.25 & 15 & 14.75 \\
\hline 10 & 4 & 27 & 23 \\
\hline
\end{tabular}

From these tables it is evident that the orthogonal case performs slightly better performing than the coaxial arrangement. In essence, one can say that there is no real difference between these two geometries. As mentioned above, the advantage of using the orthogonal case is its practical nature and ease of use.

\section{ROGOWSKI PROFILE GAP BREAKDOWN}

As mentioned, in these experiments an Nd:YAG laser was used in conjunction with a $50 \mathrm{kV}$ DC generator. This allowed larger spark gaps to be used (up to $50 \mathrm{~mm}$ ). The aim of these experiments was once more to obtain voltage breakdown levels for the Rogowski profile (uniform field) gap and to test whether there is a dependence of the breakdown voltage on the position of the laser-induced plasma along the gap axis (still using an orthogonal arrangement).

Before experiments were performed, the laser energy and pulse width across a Q-switch range of 90 to $270 \mu$ s was measured. In Figure 3 one can see the plot of energy and pulse width vs. Q-switch delay. The shaded regions represent Q-switch values for which plasma formation was not observed. 


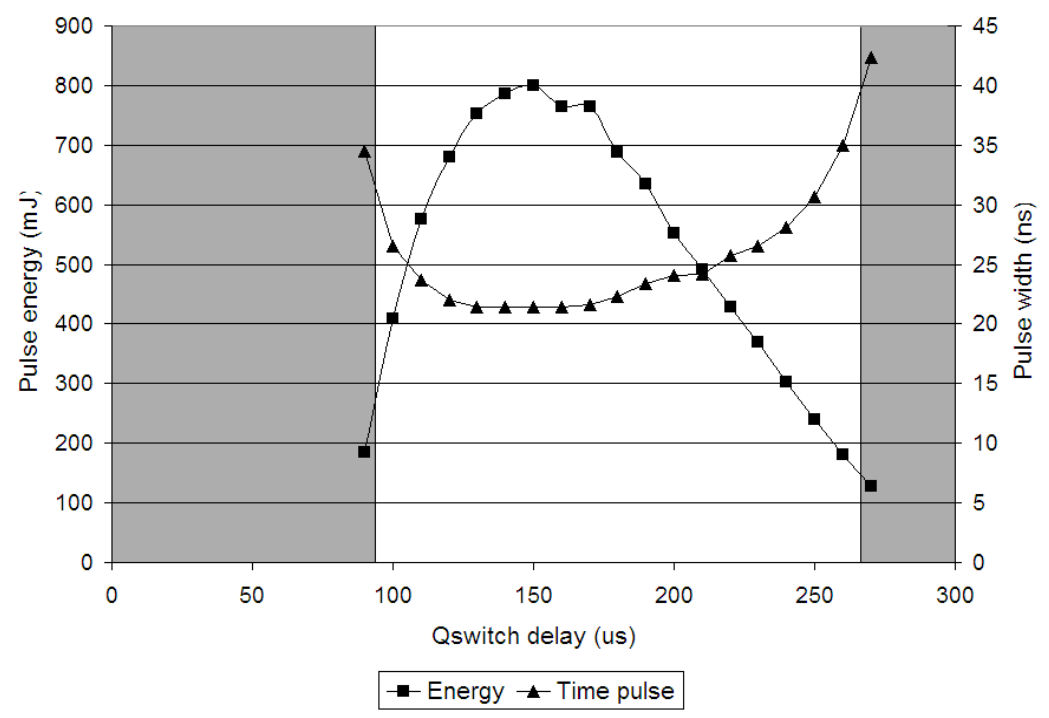

Fig. 3. Energy and pulse width vs. Q-switch delay.

\subsection{Breakdown voltage levels vs. Q-switch delay}

In this experiment, the Q-switch delay was varied from minimum to maximum and the breakdown voltage for each case was measured. This resulted in the following plot:

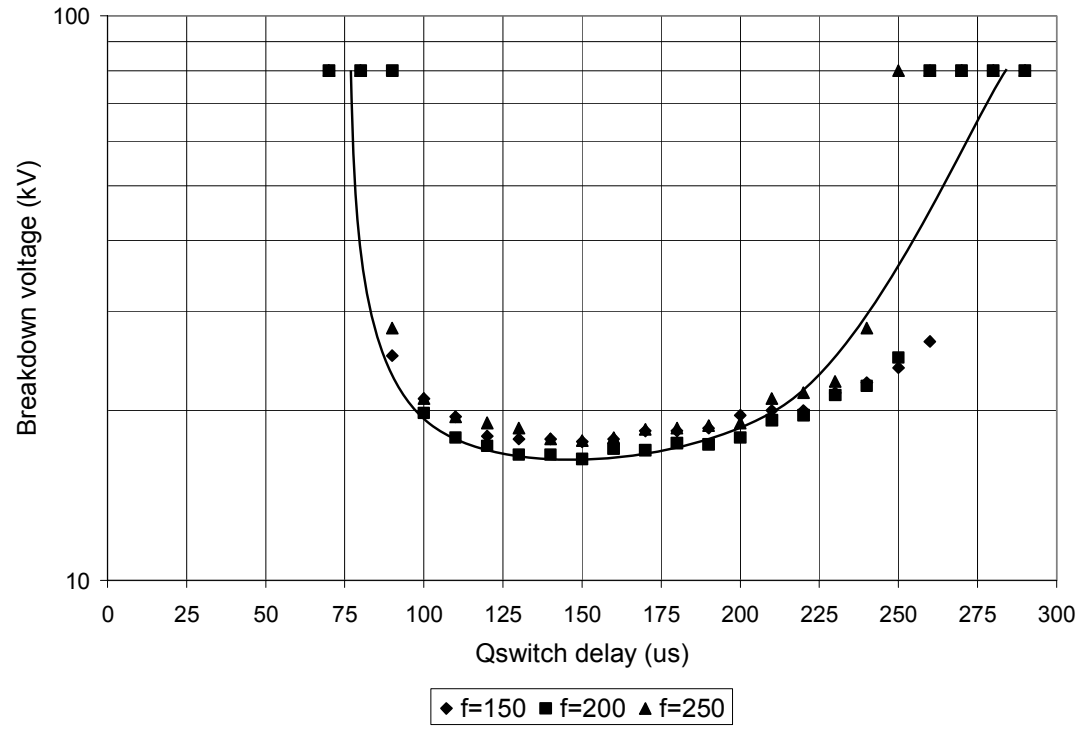

Fig. 4. Breakdown voltage vs. Q-switch delay for three different lenses (30 mm gap)

In Figure 4 it can be seen that the lowest breakdown voltage obtained is about $17 \mathrm{kV}$ and for Q-switch values of 130 to $180 \mu$ s. Another very interesting aspect of this plot is that it displays the same kind of symmetry as the plot of energy and pulse width vs. Q-switch delay. For values between 90 and $170 \mu$ s the breakdown voltage decreases sharply, whereas for values greater than $170 \mu \mathrm{s}$, the breakdown voltage increases at a much slower rate. This is expected since the breakdown voltage is linked to the peak intensity of the laser beam. In a plot of the peak intensity and the breakdown voltage as a function of Q-switch delay, the similarity between the graphs becomes evident (Figure 5). 


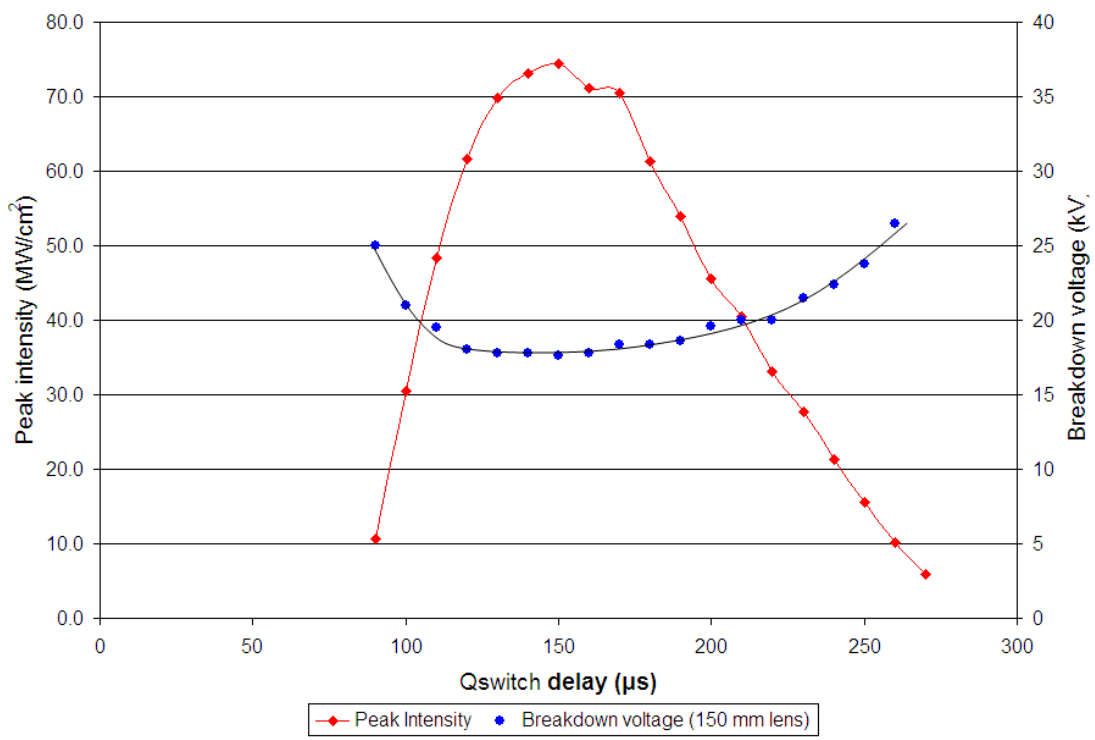

Fig. 5. Peak beam intensity and breakdown voltage as a function of Q-switch delay.

To find the breakdown voltage range of the Rogowski profile gap, the laser was set to its optimum Q-switch delay (170 $\mu \mathrm{s})$. For a $150 \mathrm{~mm}$ lens, the following results were obtained:

Table 3. Breakdown voltages at optimum Q-switch for four different gap lengths.

\begin{tabular}{|c|c|c|c|}
\hline Gap length $[\mathbf{m m}]$ & $\mathbf{V}_{\mathbf{L}}[\mathbf{k V}]$ & $\mathbf{V}_{\mathbf{W}}[\mathbf{k V}]$ & $\boldsymbol{\Delta \mathbf { V } [ \mathbf { k V } ]}$ \\
\hline 20 & 17 & 54 & 38 \\
\hline 30 & 25 & 81 & 56 \\
\hline 40 & 33 & 108 & 75 \\
\hline 50 & 40 & 135 & 95 \\
\hline
\end{tabular}

In Table 3, it is clear that the laser has a significant effect on the breakdown voltage of the gap. In fact four cases, the laser-induced breakdown voltage represents $30 \%$ of the normal withstand voltage of the gap under normal conditions. These experiments were also conducted for 3 different focal length lenses $(150,200$ and $250 \mathrm{~mm})$. It was found that the breakdown voltages only varied by $\pm 1 \mathrm{kV}$. This means that the focal length of the lens did not have a great effect on the breakdown voltage of the gap.

\subsection{Varying the position of the laser induced plasma}

So far, the experiments described involve the laser beam being focused at the centre of the gap. The question remains as to how the breakdown voltage varies with the position of the laser-induced plasma along the axis of the gap (orthogonal geometry). To perform this experiment, a height-adjustable platform (lab-jack) was used to raise or lift the spark gap so as to alter the position of the laser-induced plasma. The height was varied with millimeter accuracy from $-15 \mathrm{~mm}$ (surface of cathode) to $+15 \mathrm{~mm}$ (surface of anode). Again, 3 different lenses were used (150, 200 and $250 \mathrm{~mm})$. The following graph was obtained (Figure 6) 


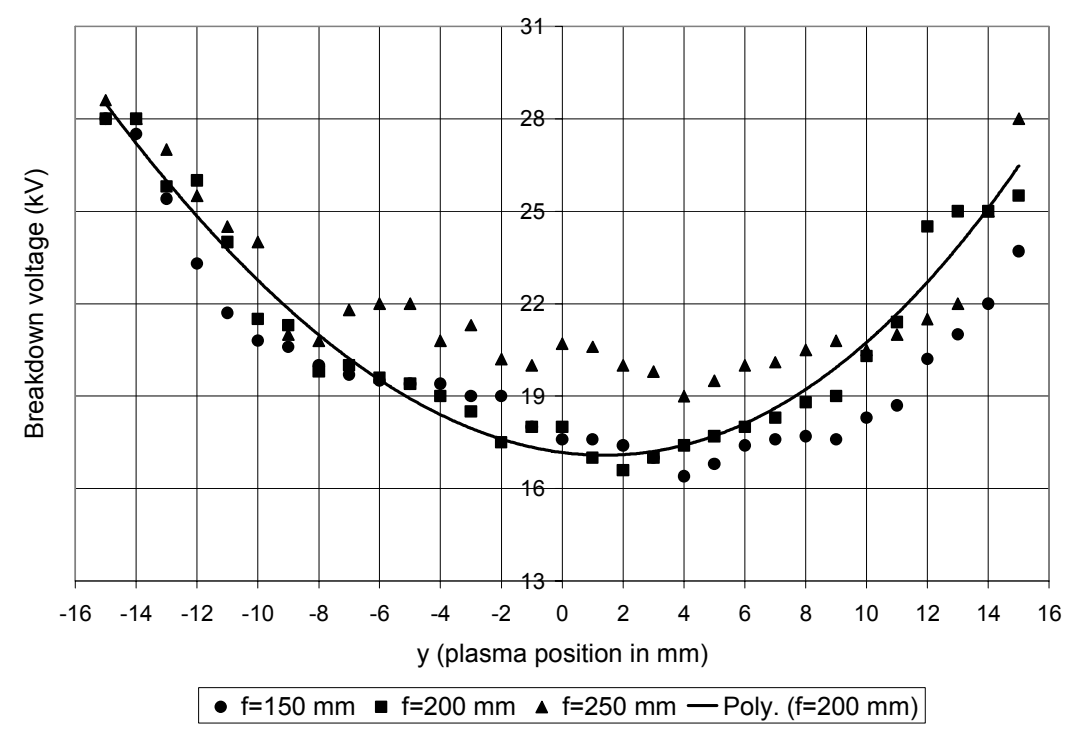

Fig. 6. Variation of breakdown voltage as a function of plasma position in the spark gap.

This graph shows a very interesting trend: The breakdown voltage at the surface of the cathode $(30 \mathrm{kV})$ is a lot higher than that at roughly the mid-point of the gap $(18 \mathrm{kV})$ and slightly higher than the breakdown voltage at the surface of the anode $(27 \mathrm{kV})$. This can be explained as follows: The laser-induced plasma provides an abundance of free electrons. Close to the cathode, the electric field does not allow these electrons to cross the gap and start the avalanche process that will lead to gap breakdown (electrons get re-absorbed by the cathode). At the surface of the anode, the free electrons are now more likely to start an avalanche that will cross the gap. The gap geometry and results can be seen in Figure 7 .
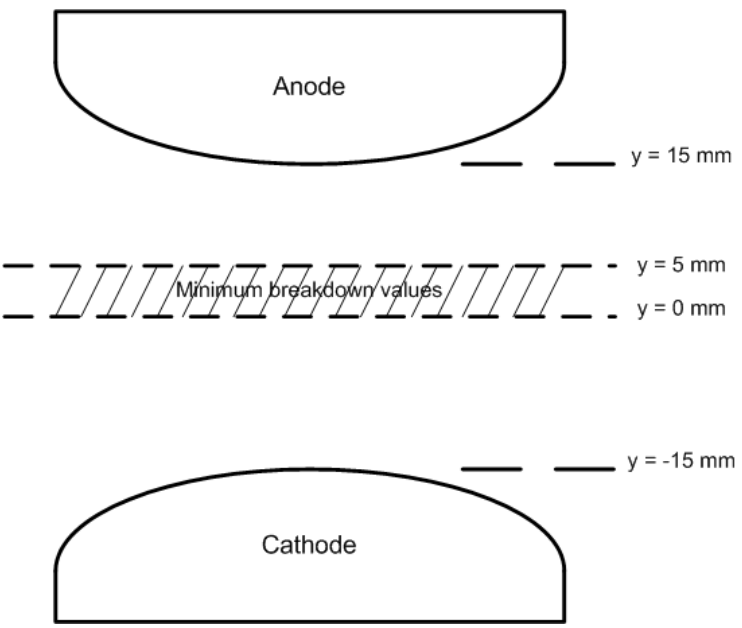

Fig. 7. Region where minimum breakdown voltage occurs.

\subsection{Shape of laser-induced arc}

During these experiments, it was noticed with the naked eye that the laser-induced arc seemed to 'avoid' the laserinduced plasma. This was a surprising observation, since it was expected that the arc would attach itself to the laserinduced plasma since the latter is by definition a conductive object. For this reason, a gated camera was used to obtain images of the laser-induced plasma and the arc in the gap and verify the preliminary observation. 
The laser-induced plasma was photographed in order to determine its shape and size. Images of a head-on and side view of the plasma were captured. These can be seen in Figure 8
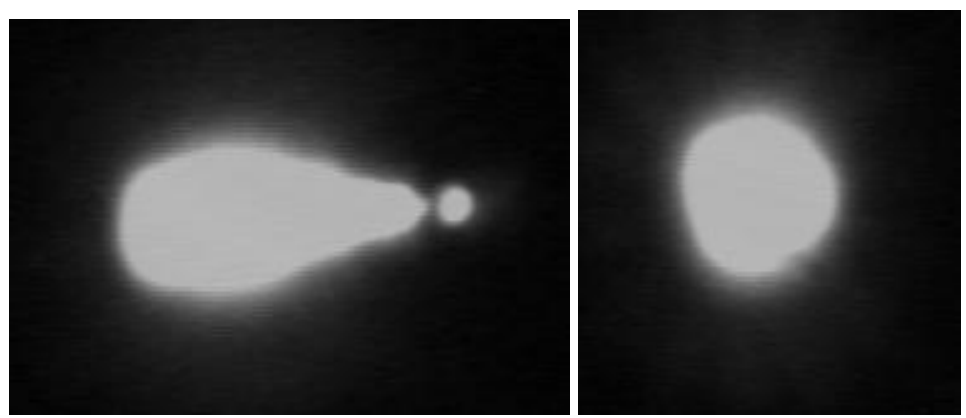

Fig. 8. Side and Head-on view of the laser-induced plasma

From these images, the size of the plasma was measured and approximated to a cylinder of length $7 \mathrm{~mm}$ and diameter 3 $\mathrm{mm}$.

An image of the arc was then captured from the head-on view. The image clearly showed that the arc avoids or simply goes around the laser-induced plasma as can be seen in Figure 9:

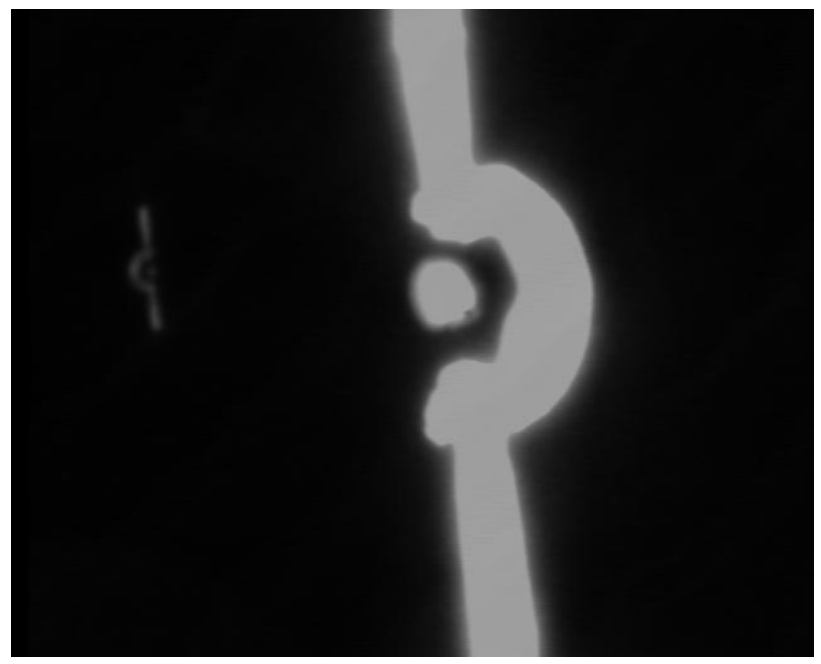

Fig. 9. Head on view of a laser-induced arc encircling the laser-induced plasma.

This shape occurred each time gap breakdown took place and was always visible in the head-on view. Whether the arc moved to the right or to the left of the laser-induced plasma was completely random. In order to investigate this phenomenon, a second camera was used. This camera used a slightly different triggering system compared to the original camera which made image capture easier.

The first step was to see how the arc shape behaved under different electric field conditions. Therefore, the gap length was set to $30 \mathrm{~mm}$ and three different voltages were used: 30,40 and $50 \mathrm{kV}$. These correspond the electric field values of $1,1.3$ and $1.7 \mathrm{kV} / \mathrm{mm}$. The images obtained show a very interesting trend (Figure 10). 

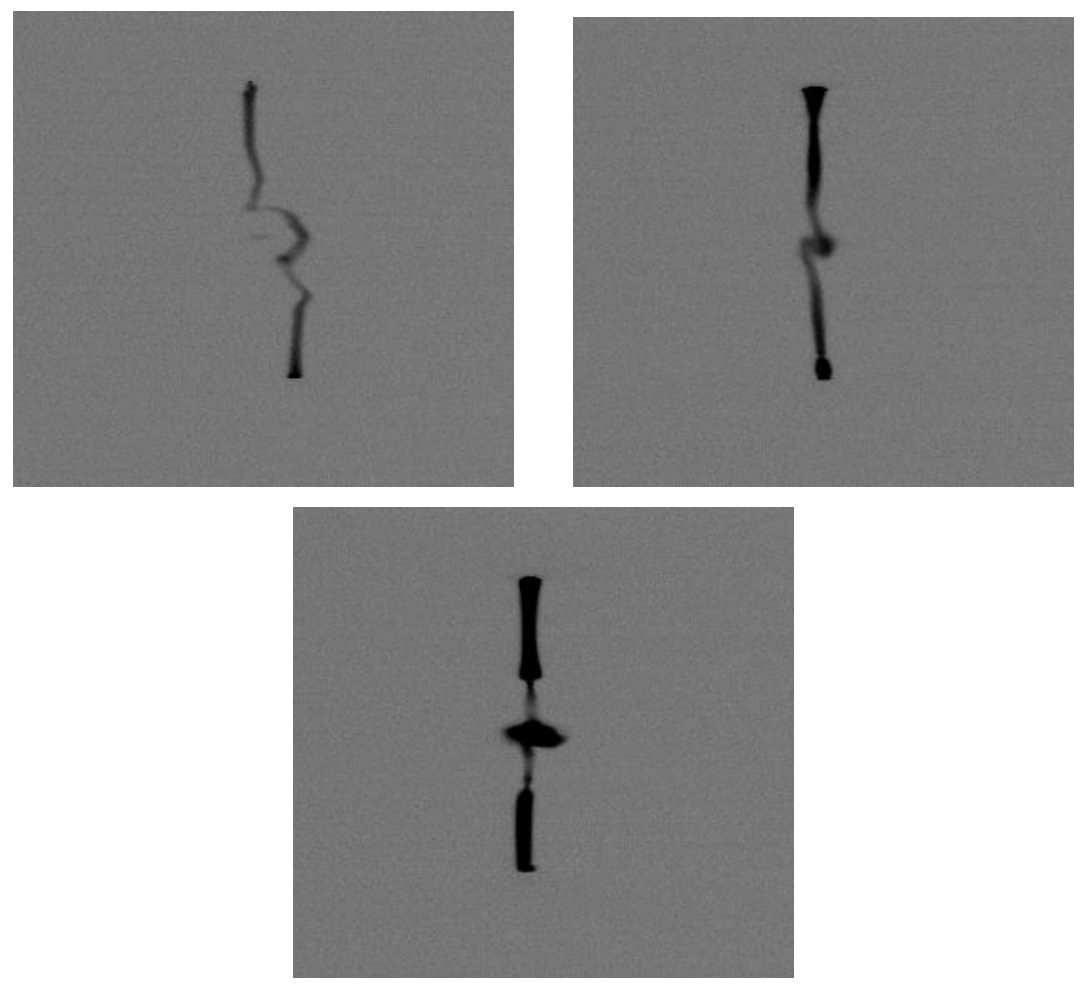

Fig. 10. Arc shapes obtained for 3 different electric field values: $1 \mathrm{kV} / \mathrm{mm}$ (top left), $1.3 \mathrm{kV} / \mathrm{mm}$ (top right) and $1.7 \mathrm{kV} / \mathrm{mm}$ (bottom)

In these images it is obvious that the electric field has a definite effect on the shape of the arc. As the field increases, so does the arc show a more orthodox tendency to move closer and eventually attach to the laser-induced plasma.

The next step was to keep the electric field constant for the three above-mentioned voltages. This meant setting up the following three scenarios: $30 \mathrm{kV} / 30 \mathrm{~mm}(1 \mathrm{kV} / \mathrm{mm}), 40 \mathrm{kV} / 40 \mathrm{~mm}(1 \mathrm{kV} / \mathrm{mm})$ and $50 \mathrm{kV} / 50 \mathrm{~mm}(1 \mathrm{kV} / \mathrm{mm})$. The objective was to see whether the arc would avoid the plasma in all three cases. The images obtained can be seen in Figure 11:

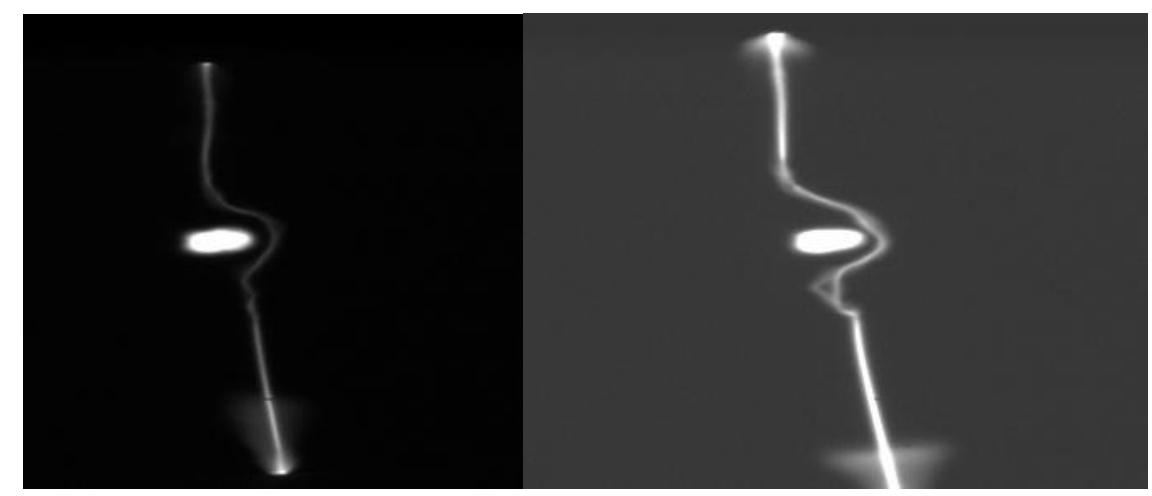

Fig. 11. Images obtained for $40 \mathrm{kV} / 40 \mathrm{~mm}$ (left) and $50 \mathrm{kV} / 50 \mathrm{~mm}$ (right) .

Figure 11 shows that when the electric field was reduced to $1 \mathrm{kV} / \mathrm{mm}$ for all cases, the arc once again avoided the laserinduced plasma. This shows that the laser-induced arc depends on a the breakdown probability of the gap. As the breakdown probability increases from the $30 \mathrm{kV} / 30 \mathrm{~mm}$ to the $50 \mathrm{kV} / 30 \mathrm{~mm}$ case, the arc moves closer to the plasma. When the breakdown probability of the latter case was reduced, (to $50 \mathrm{kV} / 50$ ) $\mathrm{mm}$, the arc tended to again move away from the plasma. 


\section{CONCLUSION}

The research presented in this paper involves an investigation into laser-induced plasmas and the role they play in lasertriggered spark gaps. Two gaps were investigated: A coaxial and an orthogonal arrangement. It was found that electrically, these two setups are similar in performance. However, the orthogonal gap proved to be more practical and versatile than the coaxial one. It was also shown that the laser beam intensity is a very important parameter when dealing with laser-triggered spark gaps. A fast rate of change of laser-beam intensity produced a very similar rate of change in breakdown voltage of the gap. The position of the laser-induced plasma also plays an important role in the breakdown process. The best results (lowest breakdown voltages) are obtained for an orthogonal arrangement when the plasma is focused close to the centre of the gap. Finally, the electric field has a definite effect on the shape of the laser-induced arc and the spatial relationship between the laser-induced plasma and the laser-induced arc. At low electric fields (and low breakdown probabilities) the arc tends to avoid the laser-induced plasma. As the electric field (and the probability of breakdown) increases so does the arc move closer (until it attaches) to the plasma.

\section{REFERENCES}

[1] Maker P. D., Terhume R. W. and C. W. Savage, "Optical third harmonic generation,” Proc. Int. Cof. Quat. Elec 1964

[2] Phuoc T. X., "An experimental and numerical study of laser-induced spark in air”, Opics and Lasers in Engineering, 43, 113-129 (2005)

[3] Steinmetz L. L., "Laser-triggered spark gap", Rev. Sci. Inst. 39 (6) (1968)

[4] Beiler M., "Laser triggered HV-switch with wide voltage range", Proc. Int. Pulsed Power Conf. 1630-1632 (1987)

[5] Megumu M., Wada A., and Shindo T., "Characteristics of upward leaders triggered by $\mathrm{CO}_{2}$ laser" in Proc. Int. Conf. on Lightning Protection (2004)

[6] Malakhov Y. I. and Rezunkov Y. A., "Lightning attachment based on laser-generated plasma channels in the atmosphere", Proc. Int. Conf. Lightning Protection, (2002)

[7] West N. J. and Jandrell I. R., "Laser-induced breakdown at 1 064, 532 and $248 \mathrm{~nm}$ in air and SF 6 ", Proc. Int. Symp. High Voltage (2005)

[8] West N. J., Jandrell I. R. and Forbes A., "Preliminary investigation into laser-high voltage interaction in the case of the streamer-to-leader process using a high-power $\mathrm{CO}_{2}$ laser", Proc. Int. Conf. Lighting Protection, 620-624 (2006) 